\title{
UN MODELO MATEMÁTICO SOBRE DINÁMICA DE VIRUS CON RESPUESTA CITOTÓXICA TIPO PRESA-PREDADOR
}

\section{A MATHEMATICAL MODEL ON VIRUS DYNAMICS WITH CTL RESPONSE PREDATOR-PREY TYPE}

\author{
Eduardo Ibargüen Mondragón, ${ }^{1},{ }^{*}$ Jhoana Patricia Romero Leitón, ${ }^{2}$ Sandra Patricia Hidalgo \\ Bonilla $^{3}$
}

Recibido:

Mayo 20 de 2014

Aceptado:

Agosto 10 de 2014

\section{Resumen}

En los últimos 20 años la modelación matemática aplicada a la inmunología (inmunología matemática) ha crecido vertiginosamente, especialmente en el estudio de dinámica poblacional a nivel celular el cual es de gran interés para la comunidad científica. En este sentido, Martin A. Nowak y Robert M. May cuentan con una amplia experiencia y han contribuido de manera significativa en este tipo de modelación. En este trabajo deseamos reconocer su trayectoria presentado el análisis cualitativo de uno de sus modelos básicos. Cabe resaltar que este análisis no aparece en el texto original publicado por ellos.

Palabras clave: ecuaciones diferenciales, análisis cualitativo, inmunología matemática.

\begin{abstract}
.
In the past 20 years mathematical modeling applied to immunology (mathematical immunology) has grown rapidly, especially in the study of population dynamics at the cellular level which is of the great interest to the scientific community. In this sense, Martin A. Nowak and Robert M. May have extensive experience and have contributed significantly in this type of modeling. In this paper we wish to acknowledge their trajectory presenting the qualitative analysis of one of their basic models. It should be noted that this analysis does not appear in the original text published by them.
\end{abstract}

Keywords: differential equations, mathematical immunology, qualitative analysis.

${ }^{1}$ Dpto. de Matemáticas y Estadística, Universidad de Nariño, edbargun@gmail.com.

* Autor corresponsal

${ }^{2}$ Instituto de Matemáticas, Universidad de Antioquia, jpatirom3@gmail.com.

${ }^{3}$ Dpto. de Química, Universidad de Nariño, sanpahil@gmail.com. 


\section{INTRODUCCIÓN}

Aunque en la actualidad la inmunología es una ciencia autónoma, sus orígenes están ligados a la microbiología. Su objeto consiste en el estudio de las respuestas de defensa que han desarrollado los seres humanos (y animales) frente a la invasión por microorganismos o partículas extrañas (Steven A. Frank, 2002). Por ejemplo, Cuando el virus de la gripa entra por la nariz o una bacteria entra por la sangre debido a un pinchazo con un clavo, se inicia un proceso infeccioso en el cual el sistema inmunológico del hospedero ataca al agente infeccioso antes de que cause daño. Este proceso se conoce como respuesta inmunológica. El uso de la modelación matemática aplicada a la inmunología ha crecido en los últimos años. En particular, en los últimos 20 años el modelamiento matemático basado en sistemas de ecuaciones diferenciales ordinarias, ecuaciones en diferencias y autómatas celulares ha incrementado considerablemente (Jane Heffernan et al., 2009; Yoram Louzoun, 2007). Los modelos matemáticos han sido utilizados en diferentes dominios de la inmunología tales como: interacción receptor antígenos, dinámica poblacional de células $B$ y células $T$, vacunación, resistencia hacia los medicamentos entre otros campos de la inmunología (Yoram Louzoun, 2007).

En el año 2000 los autores Martin A. Nowak y Robert M. May publican su libro Virus dynamics: Mathematicalprinciples of inmunology and virology en el cual presentan una serie de modelos básicos sobre la dinámica de virus. Estos modelos describen la dinámica poblacional de un proceso de infección viral en diferentes niveles cuando la respuesta inmune del hospedero reacciona a dicho proceso. Ellos describen como el virus se propaga de célula a célula, en una especie de microepidemiología que abre una perspectiva completamente nueva para la comprensión de las enfermedades infecciosas. Cabe resaltar que aunque los autores presentan el alcance y los resultados matemáticos de los diferentes modelos, no realizan el análisis cualitativo. Por esta razón, en este trabajo realizamos el análisis cualitativo del modelo denominado por los autores "respuesta citotóxica no lineal: dinámica presapredador" el cual describe una dinámica de infección básica de virus y la respuesta inmune de las células $\mathrm{T}$ en la cual, se asume a las células $\mathrm{T}$ como predadores de las células infectadas (Martin A. Nowak and Robert M. May, 2000).

\section{FORMULACIÓN DEL MODELO}

En esta sección consideramos la dinámica más simple para la interacción de una población de virus y una respuesta inmune. La dinámica básica del virus considera tres variables: el tamaño de la población de células no infectadas $(x)$; células infectadas (y); y partículas de virus libres (v). Las células no infectadas se reproducen a una tasa constante $\lambda$ y mueren a una tasa per cápita $d x$. Las partículas de virus libres infectan células no infectadas a una tasa proporcional al producto de sus poblaciones $\beta x y$. La tasa constante $\beta$, describe la eficacia de este proceso. Las células infectadas 
producen virus a una tasa per cápita $k y, \mathrm{y}$ mueren a una tasa per cápita ay. Las partículas de virus libres son removidas del sistema a una tasa per cápita $u v$.

Una vez el virus se replica, la respuesta inmune es engatillada debido al encuentro con antígenos extraños lo cual conlleva a que la respuesta citotóxica se manifieste a una tasa proporcional al producto entre la respuesta inmune $z$ y las células infectadas $y$ con constante de proporcionalidad $c$. En ausencia de estimulación la población de linfocitos citotóxicos o células $\mathrm{T}$ linfocitos decae a una tasa per cápita $b z$. Finalmente, las células infectadas son eliminadas por los linfocitos T a una tasa pyz.

La figura 1 muestra un esquema ilustrativo de la dinámica del virus y la respuesta citotóxica.

Bajo las hipótesis anteriores se obtiene el siguiente sistema de ecuaciones diferenciales:

Figura 1. Dinámica del virus y la respuesta citotóxica.
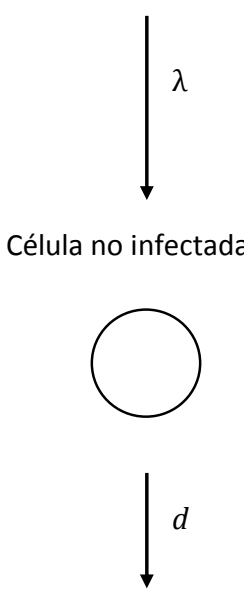

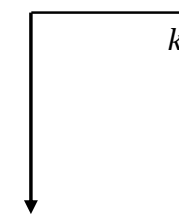

Virus libre
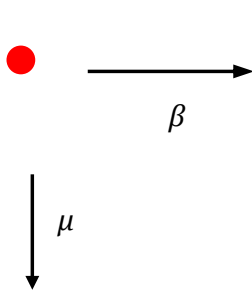

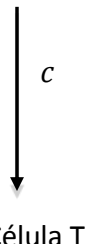

Célula infectada

Célula T
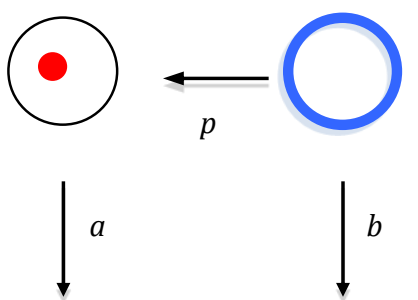

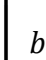

Fuente: Martin A. Nowak and Robert M. May, 'Virus dynamics: Mathematical principles of immunology and virology', Oxford UniversityPress, New York, 2000. p 18. (Modificado). 


$$
\begin{aligned}
& \frac{d x}{d t}=\lambda-d x-\beta x v \\
& \frac{d y}{d t}=\beta x v-a y-p y z \\
& \frac{d v}{d t}=k y-u v \\
& \frac{d z}{d t}=c y z-b z
\end{aligned}
$$

\section{ANALISIS CUALITATIVO}

En esta sección se determina tanto la existencia como la estabilidad de las soluciones de equilibrio del sistema (1).

Las soluciones de equilibrio están determinadas por las soluciones del sistema algebraico que se obtiene de igualar la parte del lado derecho del sistema de ecuaciones diferenciales (1) a cero. En este caso estas soluciones de equilibrio o estados estacionarios son

$$
\begin{aligned}
& \bar{P}_{0}=(\lambda / d, 0,0,0) \\
& \bar{P}_{1}=\left(\frac{a v}{\beta k}, \frac{\beta \lambda k-d a \mu}{\beta a k}, \frac{\beta \lambda k-d a \mu}{\beta a \mu}, 0\right) \\
& \bar{P}_{2}=\left(\frac{\lambda c \mu}{c d \mu+\beta b k}, \frac{b}{c}, \frac{\beta k}{c \mu}, \frac{1}{p}\left(\frac{\lambda \beta c k}{c d \mu+\beta b k}-a\right)\right)
\end{aligned}
$$

Debido a que se está modelando una dinámica poblacional el conjunto de interés biológico se establece como

$$
\Omega=\left\{\left(x_{1}, x_{2}, x_{3}, x_{4}\right) \in R^{4}: x_{i} \geq 0 \text { para } i=1,2,3,4\right\}
$$

En este sentido $P_{0}$ pertenece a $\Omega$, sin embargo $P_{1}$ y $P_{2}$ necesitan condiciones para pertenecer a $\Omega$ las cuales serán establecidas mas adelante. A continuación se determinará la estabilidad del equilibrio libre de infección $P_{0}$. Para este fin observemos que la matriz jacobiana de (1) está dada por

$$
J\left(\begin{array}{l}
x \\
y \\
v \\
z
\end{array}\right)=\left(\begin{array}{cccc}
-(d+\beta v) & 0 & -\beta x & 0 \\
\beta v & -(a+p z) & \beta x & -p y \\
0 & k & -\mu & 0 \\
0 & c z & 0 & c y-b
\end{array}\right)
$$

Luego, el Jacobiano evaluado en $P_{0}$ está dado por

$$
J\left(\bar{P}_{0}\right)=\left(\begin{array}{cccc}
-d & 0 & -\frac{\beta \lambda}{d} & 0 \\
0 & -a & \frac{\beta \lambda}{d} & 0 \\
0 & k & -\mu & 0 \\
0 & 0 & 0 & -b
\end{array}\right)
$$

Obsérvese que los valores propios de $J\left(P_{0}\right)$ son $\lambda_{1}=-d, \lambda_{2}=-b$ y las raíces de la ecuación cuadrática

$$
\lambda^{2}+(a+\mu) \lambda+a \mu\left(1-R_{0}\right)=0,
$$

donde

$$
R_{0}=\frac{\beta \lambda k}{a d \mu}
$$

Es el número reproductivo básico de la infección el cual se interpreta como el número de nuevas células infectadas producidas por una célula infectada cuando la mayoría de células no están infectadas.

Se verifica que las soluciones de la anterior ecuación cuadrática tienen parte real 
negativa cuando $R_{0}<1$, lo cual implica que el equilibrio libre de infección $P_{0}$ es localmente asintóticamente estable (Lawrance Perko, 2000). Por otro lado, en términos de $R_{0}$ el equilibrio $P_{1}$ se reescribe como $P_{1}=\left(\bar{x}_{1}, \bar{y}_{1}, \bar{v}_{1}, 0\right)$ donde

$$
\begin{aligned}
& \bar{x}_{1}=\frac{x_{0}}{R_{0}}, \quad \bar{y}_{1}=\left(R_{0}-1\right) \frac{d \mu}{\beta k}, \\
& \bar{v}_{1}=\left(R_{0}-1\right) \frac{d}{\beta}
\end{aligned}
$$

En consecuencia, el equilibrio $P_{1}$ tiene sentido biológico cuando $R_{0}>1$. Ahora, el Jacobiano de (1) evaluado en $P_{1}$ está dado por

$$
\begin{aligned}
& J\left(\bar{P}_{1}\right)=\left(\begin{array}{cccc}
-\left(d+\beta \bar{v}_{1}\right) & 0 & -\beta \bar{x}_{1} & 0 \\
\beta \bar{v}_{1} & -a & \beta \bar{x}_{1} & -p \bar{y}_{1} \\
0 & k & -\mu & 0 \\
0 & 0 & 0 & c \bar{y}_{1}-b
\end{array}\right) \\
& J\left(\bar{P}_{0}\right)=\left(\begin{array}{cccc}
-\frac{\lambda}{\bar{x}_{1}} & 0 & -\beta \bar{x}_{1} & 0 \\
\beta \bar{v}_{1} & -a & \beta \bar{x}_{1} & -p \bar{y}_{1} \\
0 & k & -\mu & 0 \\
0 & 0 & 0 & c \bar{y}_{1}-b
\end{array}\right)
\end{aligned}
$$

Cuyos valores propios son

$$
\begin{aligned}
\eta_{1} & =c \bar{y}_{1}-b \\
& =\left(R_{\mathrm{o}}-1-\frac{b \beta k}{c d \mu}\right) \frac{c d \mu}{\beta k}
\end{aligned}
$$

y las raíces de

$$
\eta^{3}+a_{1} \eta^{2}+a_{2} \eta+a_{3}=0
$$

donde

$$
\begin{aligned}
& a_{1}=\frac{\lambda}{\bar{x}_{1}}+a+\mu \\
& a_{2}=\frac{\lambda}{\bar{x}_{1}}(a+\mu) \\
& a_{3}=\beta \overline{x_{1}} \beta \bar{v}_{1} k
\end{aligned}
$$

En consecuencia, $\eta_{1}<0 \eta_{1}<0 \quad$ si y solo si

$$
R_{\mathrm{o}}<1+\frac{b \beta k}{c d \mu}
$$

Por otro lado, a partir del criterio de RouthHurwitz se establece que las raíces de la anterior ecuación cúbica tienen parte real negativa si $a_{1}, a_{2}, a_{3}, \mathrm{y} a_{1} a_{2}-a_{3}>0$. Es claro que $a_{1}>0$ y $a_{2}>0$ mientras que $a_{3}>0$ y solo si $R_{0}>1$. Además,

$$
\begin{aligned}
& \delta_{3}=a_{1} a_{2}-a_{3} \\
& =\left(\frac{\lambda}{\bar{x}_{1}}\right)^{2}(a+\mu)+\beta \lambda k\left(a^{2}+a \mu+\mu^{2}\right)+d a \mu>0
\end{aligned}
$$

Por lo tanto, el equilibrio $P_{1}$ es localmente asintóticamente estable si se satisface la condición (2).

Obsérvese que $P_{2}$ tiene sentido biológico si

$$
\frac{\lambda \beta c k}{c d \mu+\beta b k}-a>0,
$$

de donde obtenemos la condición de existencia

$$
R_{\mathrm{O}}>1+\frac{b \beta k}{c d \mu}
$$

Repitiendo un proceso similar al utilizado para la prueba de estabilidad del equilibrio $P_{1}$ se verifica la estabilidad asintótica del equilibrio $P_{2}$. 
En la siguiente tabla se presenta un resumen de existencia y estabilidad de las soluciones de equilibrio.

Tabla 1. Resumen de existencia y estabilidad.

\begin{tabular}{|l|l|l|}
\hline Equilibrio & Existencia & Estabilidad \\
\hline $\bar{P}_{0}$ & Siempre & $R_{0}<1$ \\
$\bar{P}_{1}$ & $R_{0}>1$ & $1<R_{0}<1+\frac{b \beta k}{c d \mu}$ \\
$\bar{P}_{2}$ & $R_{0}>1+\frac{b \beta k}{c d \mu}$. & Estable \\
\hline
\end{tabular}

Fuente: Esta investigación.

Cuando el número reproductivo básico de la infección es menor que uno $\left(R_{0}<1\right)$ la infección es eliminada, es decir, la carga viral inicial no es capaz de reproducirse y por lo tanto es eliminada. Mientras que cuando el número reproductivo básico de la infección es mayor que uno $\left(R_{0}>1\right)$, la infección progresa en los siguientes escenarios:

Cuando

$$
1<R_{\mathrm{O}}<1+\frac{b \beta k}{c d \mu}
$$

infección progresa sin activar la respuesta citotóxica.

Cuando $\quad R_{0}>1+\frac{b \beta k}{c d \mu} \quad$ la infección progresa activando la respuesta citotóxica.

\section{CONCLUSIONES}

En este artículo se formula y analiza un modelo matemático básico que describe la dinámica de infección del virus y el efecto de una respuesta inmune de tipo citotóxica. Este tipo de respuesta se presenta en la respuesta inmunológica celular la cual es la principal respuesta en enfermedades adquiridas por virus tales como el VIHSIDA, influenza, fiebre amarilla, entre otras enfermedades.

\section{AGRADECIMIENTOS}

E. Ibarguen agradece el apoyo recibido del proyecto número 082-16/08/2013 (VIPRIUDENAR)

\section{BIBLIOGRAFÍA}

Jane Heffernan, Beni Sahai, Robert Smith, 'Mathematical Immunology of Infectious Diseases'. May 17, 2009, preprint.

Lawrance Perko, 'DifferentialEquations and DynamicalSystems', Springer-Verlag, New York, 1991.

Martin A. Nowak and Robert M. May, 'Virus dynamics: Mathematical principles of immunology and virology', Oxford UniversityPress, New York, 2000.

Steven A. Frank, 'Immunology and Evolution of Infectious Desease', Princeton UniversityPress, New Jersey, 2002.

Yoram Louzoun, 'The evolution of mathematical immunology', Immunological Reviews 2007 Vol. 216: 9-20, 2007. 